\title{
Generalized L-Spline Wavelet Bases
}

\author{
Ildar Khalidov, Thierry Blu, Michael Unser \\ Biomedical Imaging Group, Ecole Polytechnique Fédérale de Lausanne (EPFL), Lausanne, \\ Switzerland
}

\begin{abstract}
We build wavelet-like functions based on a parametrized family of pseudo-differential operators $\mathrm{L}_{\vec{\nu}}$ that satisfy some admissibility and scalability conditions. The shifts of the generalized B-splines, which are localized versions of the Green function of $\mathrm{L}_{\vec{\nu}}$, generate a family of L-spline spaces. These spaces have the approximation order equal to the order of the underlying operator. A sequence of embedded spaces is obtained by choosing a dyadic scale progression $a=2^{i}$. The consecutive inclusion of the spaces yields the refinement equation, where the scaling filter depends on scale. The generalized L-wavelets are then constructed as basis functions for the orthogonal complements of spline spaces. The vanishing moment property of conventional wavelets is generalized to the vanishing null space element property. In spite of the scale dependence of the filters, the wavelet decomposition can be performed using an adapted version of Mallat's filterbank algorithm.
\end{abstract}

Keywords: Wavelets, splines, differential operators, Green's functions, continuous-time signal processing, multiresolution approximation, multiresolution analysis

\section{INTRODUCTION}

The wavelet transform is widely used nowadays to perform multiresolution signal analysis. ${ }^{1}$ It has applications in numerous areas of applied mathematics such as signal processing, compression, denoising, to mention but a few. Over the years, many wavelet bases have been designed; they were typically specified to satisfy certain properties, such as vanishing moments, approximation order, compact support etc.

It is well known that the analysis wavelet behaves like a multi-scale version of the derivative operator of the corresponding order; this feature is intimately related to the vanishing moment and approximation order properties. $^{1}$ Interestingly, it is the B-spline component that is present within every scaling function that induces this special behavior; the B-spline is fundamental in this respect because it generates the same space as the Green function of the differential operator. ${ }^{2}$

In this work we generalize the construction of wavelets starting from an arbitrary family of pseudo-differential operators $\mathrm{L}_{\vec{\nu}}$, satisfying certain admissibility and scalability conditions. The L-spline spaces spanned by dyadic shifts of the Green functions of $\mathrm{L}_{\vec{\nu}}$ yield a multiresolution analysis. The approximation order turns out to be equal to the order of the operator $\mathrm{L}_{\vec{\nu}}$. The resulting wavelets are non-stationary, which means that the basis functions at the different scales are no longer dilates of one another; however, the wavelet decomposition can still be performed using a modification of Mallat's filterbank algorithm with precomputed filters for each scale.

The resulting class of L-spline wavelets is quite large; it comprises the standard spline wavelets, which correspond to the choice $\mathrm{L}=\mathrm{D}^{N}$, where $\mathrm{D}$ is a derivative. Also included are the fractional wavelets, ${ }^{3}$ as well as the generalized exponential B-spline wavelets. $^{4,5}$ Even though our construction is restricted to time-invariant operators, we can consider rather general transfer functions, which are characterized by their order $r$ of growth.

This paper is organized as follows. In Section 2, we introduce the key notions that are used throughout the paper. In Section 3, we specify L-spline multiresolution analyses using generalized B-splines as basis functions. In Section 4, we introduce the L-spline wavelets; we then study their properties in Section 5. Finally, we discuss the implementation of the decomposition-reconstruction algorithm and study an example illustrating the concept.

Further author information: (ildar.khalidov, thierry.blu, michael.unser)@epfl.ch 


\section{PRELIMINARIES}

In order to simplify our derivations, we introduce the scaling operator $S_{a}: f(\cdot) \rightarrow f(\cdot / a)$ and the translation operator $T_{k}: f(\cdot) \rightarrow f(\cdot-k)$. They satisfy the relation $S_{a} T_{k}=T_{a k} S_{a} . \mathcal{F}_{\alpha}$ is the generalized Fourier operator $\mathcal{F}_{\alpha}: f(\cdot) \rightarrow \int_{-\infty}^{\infty} f(t) e^{-(\alpha+j \omega) t} \mathrm{~d} t, \alpha \in \mathbb{C}$. The traditional Fourier operator $\mathcal{F}$ is a particular case of this definition: namely, $\mathcal{F}=\mathcal{F}_{0}$.

Let us consider a linear, shift-invariant operator L. This operator is characterized by its Laplace transform $L(s)$ or, equivalently, by its frequency response $\hat{L}(\omega)=L(j \omega)$.

Definition 2.1. L is of order $r$ if and only if, for all positive $\rho<r-1 / 2$, we have that

$$
\sum_{n \in \mathbb{Z}} \frac{|\omega+2 \pi n|^{2 \rho}}{1+|\hat{L}(\omega+2 \pi n)|^{2}} \leq C_{\rho}<\infty
$$

Essentially, Definition 2.1 requires the frequency response $\hat{L}(\omega)$ of the operator to grow at the rate of $\omega^{r}$ as $\omega \rightarrow \infty$.

Definition 2.2. L is spline-admissible of order $r$ if and only if the following conditions are satisfied ${ }^{6}$ :

1. L is a linear shift-invariant operator of smoothness order $r>1 / 2$;

2. $\mathrm{L}$ has a well-defined inverse $\mathrm{L}^{-1}$ with impulse response $\rho \in S^{\prime}$. Thus, $\rho$ is a Green function of $\mathrm{L}: \mathrm{L}\{\rho\}=\delta$;

3. There exists a localization operator $\Delta=\sum_{k \in \mathbb{Z}} d[k] T_{k}$ with $d \in \ell_{1}$ such that the corresponding generalized $B$-spline $\beta=\Delta\{\rho\}$ satisfies the Riesz basis condition.

DEFINITION 2.3. Let $\mathrm{L}$ be a spline-admissible operator of order $r$ and let $\beta=\Delta\{\rho\}$ be a corresponding B-spline. $W e$ say that the generalized B-spline $\beta(t)$ is properly localized if, for any zero $\alpha$ of the transfer function $L(s)$, $\Delta(s)$ has a zero of the same order at $s=\alpha$.

The localization process in 3 ) is illustrated in Figure 1 for the first-order differential operator $\hat{L}_{\alpha}(\omega)=j \omega-\alpha$. The Green function, which is a causal exponential, is truncated by subtracting its weighted and shifted version; this corresponds to the choice $\Delta=\mathrm{I}-e^{a \alpha} T_{a}$. Note that, for $\alpha<0$, the Green function $\rho_{\alpha}$ itself may be considered as a B-spline; however, the proper-localization condition is not met in this case.

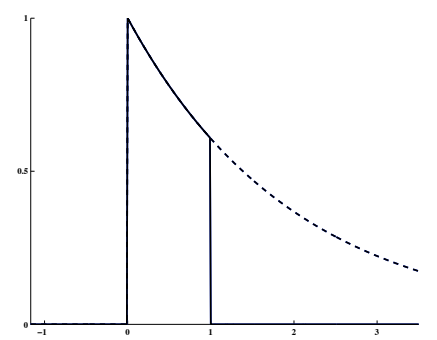

Figure 1. Localization of the Green function for $a=1$.

Definition 2.4. A family of operators $\mathrm{L}_{\vec{\nu}}$ depending on some parameters $\vec{\nu}$ is called pseudo-scale invariant if for all $a>0$ it satisfies

$$
S_{a} \mathrm{~L}_{a \vec{\nu}}=c(a) \mathrm{L}_{\vec{\nu}} S_{a},
$$

where $c(a)$ is an arbitrary function of $a$.

The equivalent definition in the Fourier domain,

$$
\hat{\mathrm{L}}_{a \vec{\nu}}=c(a) S_{a} \hat{\mathrm{L}}_{\vec{\nu}}
$$


is obtained by applying the Fourier operator $\mathcal{F}$ on both sides of (1) and using the relation $\mathcal{F} S_{a}=|a| S_{1 / a} \mathcal{F}$.

For the Green function $\rho_{a \vec{\nu}}$ of a pseudo-scale invariant operator $\mathrm{L}_{a \vec{\nu}}$, we have $\mathrm{L}_{a \vec{\nu}} \rho_{a \vec{\nu}}=\delta$. By applying $S_{a}$ on both sides, we obtain $c(a) \mathrm{L}_{\vec{\nu}} S_{a} \rho_{a \vec{\nu}}=|a| \delta$; thus, the following scaling relation is satisfied:

$$
\rho_{\vec{\nu}}=\frac{c(a)}{|a|} S_{a} \rho_{a \vec{\nu}}
$$

EXAMPLE 1. Consider the generalized fractional differential operator $\mathrm{L}_{\alpha}^{\gamma}$ of order $\gamma>\frac{1}{2}: \hat{\mathrm{L}}_{\alpha}^{\gamma}(\omega)=(j \omega-\alpha)^{\gamma}$. Here $\vec{\nu}=\alpha \in \mathbb{C}$ is a complex parameter. We readily check that the condition (2) is met:

$$
\hat{\mathrm{L}}_{a \vec{\nu}}^{\gamma}=a^{\gamma}\left(j \frac{\omega}{a}-\alpha\right)^{\gamma}=a^{\gamma} S_{a} \hat{\mathrm{L}}_{\vec{\nu}}^{\gamma} .
$$

We therefore see that $\mathrm{L}_{\alpha}^{\gamma}$ is pseudo-scale invariant with $c(a)=a^{\gamma}$.

\section{L-SPLINE MULTIRESOLUTION}

A generalized L-spline $s(t)$ for the pseudo-scale invariant, spline-admissible operator $\mathrm{L}_{\vec{\nu}}$ with equally spaced knots $t_{k}=a k, a>0$, is a function that satisfies ${ }^{6}$

$$
\mathrm{L}_{\vec{\nu}} s(t)=\sum_{k \in \mathbb{Z}} a[k] \delta(t-a k) .
$$

By applying the inverse operator $\mathrm{L}_{\vec{\nu}}^{-1}$, we get

$$
s(t)=\sum_{k \in \mathbb{Z}} a[k] \rho_{\vec{\nu}}(t-a k)+p_{\vec{\nu}}(t),
$$

where $p_{\vec{\nu}}(t)$ is a solution of the homogeneous equation $\mathrm{L}_{\vec{\nu}}\left\{p_{\vec{\nu}}\right\}=0$.

Let $\mathrm{L}_{\vec{\nu}}$ be a pseudo-scale invariant, spline-admissible operator of order $r$ with localization operator $\Delta_{\vec{\nu}}$ for each $\vec{\nu}$; let $\beta_{\vec{\nu}}=\Delta_{\vec{\nu}} \rho_{\vec{\nu}}$ be a properly localized B-spline. We then define the B-spline at scale $a>0$ as $\beta_{\vec{\nu}, a}=$ $S_{a} \beta_{a \vec{\nu}}=\sum_{k \in \mathbb{Z}} S_{a} d_{a \vec{\nu}}[k] T_{k} \rho_{a \vec{\nu}}=\left(\sum_{k \in \mathbb{Z}} d_{a \vec{\nu}}[k] T_{a k} \rho_{\vec{\nu}}\right) \cdot a / c(a)$. The corresponding scaled localization operator $\Delta_{\vec{\nu}, a}$ is given by $\Delta_{\vec{\nu}, a}=\sum_{k \in \mathbb{Z}} d_{a \vec{\nu}}[k] T_{a k}$.

Next, we consider the space $V_{a}$ spanned by the shifted B-splines, $V_{a}=\left\{s(t): s(t)=\sum_{k \in \mathbb{Z}} c[k] \beta_{\vec{\nu}, a}(t-\right.$ $\left.a k), c[k] \in l_{2}\right\}$. From (3), we see that any element of $V_{a}$ is an L-spline. To demonstrate that any squaresummable L-spline $s(t)$ at scale $a$ belongs to $V_{a}$, we prove the two following propositions.

Proposition 1. Any function $s(t)=\sum_{k \in \mathbb{Z}} a[k] \rho_{\vec{\nu}}(t-a k) \in L_{2}$ belongs to $V_{a}$.

Proof. We have that

$$
\int_{0}^{2 \pi} \frac{\left|A\left(e^{j \omega a}\right)\right|^{2}}{\left|\hat{\Delta}_{\vec{\nu}, a}(\omega)\right|^{2}} \sum_{k \in \mathbb{Z}} \frac{\left|\hat{\Delta}_{\vec{\nu}, a}(\omega)\right|^{2}}{|\hat{L}(\omega a+2 \pi k j)|^{2}} \mathrm{~d} \omega=\int_{-\infty}^{\infty}|\hat{s}(\omega)|^{2} \mathrm{~d} \omega<\infty
$$

where the inner sum on the left-hand side can be estimated using the lower Riesz bound for $\beta_{\vec{\nu}, a}$. Therefore, $s(t)=\sum_{k \in \mathbb{Z}} c[k] \beta_{\vec{\nu}, a}(t-a k)$ with $C\left(e^{j \omega a}\right)=A\left(e^{j \omega a}\right) / \hat{\Delta}_{\vec{\nu}, a}(\omega)$ and $c[k] \in l_{2}$.

This proposition allows us to express the Green-function part of an arbitrary L-spline with the help of shifted B-splines. We will now show that the null-space element can also be represented as a weighted sum of B-spline basis functions. In order to do so, we introduce the generalized Strang-Fix conditions for a scaling function $\varphi$ and an operator $\mathrm{L}$.

Definition 3.1. The scaling function $\varphi$ satisfies the generalized Strang-Fix conditions for a given operator $\mathrm{L}$ if, for every $\alpha$ such that $L(s)$ has a zero of some order $\gamma$ at $s=\alpha, \mathcal{F}_{\alpha}\{\varphi\}(\omega)$ has a zero of order $\gamma$ at $\omega=2 \pi k$ for all $k \in \mathbb{Z}, k \neq 0$, and $\mathcal{F}_{\alpha}\{\varphi\}(0)=c_{\alpha} \neq 0$. 
Using Poisson's summation formula, one can demonstrate that the above generalized Strang-Fix conditions for the operator $\mathrm{L}$ are sufficient for the null-space reconstruction property: For every $p(t)$ such that $\mathrm{L}\{p(t)\}=0$, there exist $p[k]$ such that

$$
p(t)=\sum p[k] \varphi(t-k)
$$

We now have all the ingredients to prove the announced result.

Proposition 2. Let $L_{\vec{\nu}}(s)$ satisfy $L_{\vec{\nu}}(\alpha+2 \pi k j / a) \neq 0$ for each $\alpha$ such that $L_{\vec{\nu}}(\alpha)=0$ and for all $k \in \mathbb{Z}$. In this case, any function $p_{\vec{\nu}}$ such that $\mathrm{L}_{\vec{\nu}}\left\{p_{\vec{\nu}}\right\}=0$ can be expressed via the shifted $B$-splines $\left\{\beta_{\vec{\nu}, a}(\cdot-a k)\right\}_{k \in \mathbb{Z}}$.

Proof. It suffices to demonstrate that $\beta_{\vec{\nu}, a}$ satisfies the generalized Strang-Fix conditions for $\mathrm{L}_{\vec{\nu}}$. In order for $\beta_{\vec{\nu}, a}$ to meet the proper-localization condition, the transfer function $\Delta_{\vec{\nu}, a}(s)$ must behave as $|s-\alpha|^{\gamma}$ as $s \rightarrow \alpha$, for all $\alpha$ being $\gamma$-order zeros of $\mathrm{L}_{\vec{\nu}}$. As $L_{\vec{\nu}}(\alpha+2 \pi k j / a) \neq 0, k \in \mathbb{Z}$, the $2 \pi j / a$-periodicity of $\Delta_{\vec{\nu}, a}(s)$ yields the desired result.

We have proved that for any generalized L-spline $s(t) \in L_{2}$ there exist $c[k]$ such that

$$
s(t)=\sum_{k=-\infty}^{+\infty} c[k] \beta_{\vec{\nu}, a}(t-a k),
$$

This ensures that the generalized B-splines form a complete basis of the L-spline space.

We now consider the dyadic scales $a=2^{i}$ and define $V_{(i)}=V_{2^{i}}=\left\{s_{i}(t)=\sum_{k \in \mathbb{Z}} c_{k} \varphi_{i}\left(t-2^{i} k\right): c \in l_{2}\right\}$, where $\varphi_{i}(t)=\beta_{2^{i}}(t) /\left\|\beta_{2^{i}}\right\|_{L_{2}}$ is the normalized scaling function.

We give in Figure 2 a diagram that summarizes the construction of the L-spline spaces.

$$
\begin{gathered}
L_{\vec{\nu}} V_{a}=\operatorname{span}\{\delta(\cdot-a k)\} \\
L_{\vec{\nu}} \uparrow \sqrt{ } H_{\vec{\nu}}=L_{\vec{\nu}}^{-1} \\
V_{a}=\operatorname{span}\left\{\rho_{\vec{\nu}}(\cdot-a k)\right\} \\
\Delta_{\vec{\nu}, a}^{-1} \uparrow \sqrt{ } \Delta_{\vec{\nu}, a} \\
V_{a}=\operatorname{span}\left\{\beta_{\vec{\nu}, a}(\cdot-a k)\right\}
\end{gathered}
$$

Figure 2. L-spline space-construction process

As seen from (3), the space $V_{(i)}$ is included in $V_{(i-1)}$. We therefore have a ladder of spaces

$$
\cdots \subset V_{(i+1)} \subset V_{(i)} \subset V_{(i-1)} \subset \ldots
$$

The following proposition gives a result on the approximation error decay rate in $V_{a}$ as the scale gets finer.

Proposition 3. Let $P_{a}$ be the orthogonal projector into the space $V_{a}$. Then, for every $f \in L_{2}$ such that $L_{\vec{\nu}} f \in L_{2},\left\|f-P_{a} f\right\|_{L_{2}}=O\left(a^{r}\right)$ as $a \rightarrow 0$.

The proof is quite technical and will be published elsewhere. As a consequence of this proposition, we can prove that $\bigcup V_{(i)}$ is dense in $L_{2}$. 


\section{MULTIRESOLUTION BASIS FUNCTIONS}

The function $\varphi_{i+1} \in V_{(i+1)} \subset V_{(i)}$ can be expressed as

$$
\varphi_{i+1}(t)=\sum_{k} h_{i}[k] \varphi_{i}\left(t-2^{i} k\right),
$$

where the filter $h_{i} \in l_{2}$ because $\varphi_{i+1} \in L_{2}$ and $\varphi_{i}$ generates a Riesz basis. The frequency response $H_{i}\left(e^{j 2^{i} \omega}\right)$ is given by

$$
H_{i}\left(e^{j 2^{i} \omega}\right)=c_{i} \cdot \frac{\hat{\Delta}_{\vec{\nu}, 2^{i+1}}(\omega)}{\hat{\Delta}_{\vec{\nu}, 2^{i}}(\omega)},
$$

where $c_{i}$ is a normalization constant. We observe that it is possible to tune the properties of the scaling filter by choosing the family of localization operators $\Delta_{\vec{\nu}}$ appropriately. For the case of the E-spline wavelets, where $L(s)$ is a rational function, the localization operator $\Delta_{\vec{\nu}, a}$ consists of the factors $\left(1-e^{\left(\alpha_{k}-j \omega\right) a}\right)^{m_{k}}$ corresponding to zeros $\alpha_{k}$ of multiplicity $m_{k}$ of $L(s)$. The scaling filter $H_{i}\left(e^{j \omega a}\right)$ is therefore a product of the filters $\left(1+e^{\left(\alpha_{k}-j \omega\right) a}\right)^{m_{k}}$ for all $k .^{4}$

The dual scaling function is defined by

$$
\hat{\tilde{\varphi}}_{i}(\omega)=\hat{\varphi}_{i}(\omega) / A_{i}\left(e^{j 2^{i} \omega}\right)
$$

with $A_{i}\left(e^{j 2^{i} \omega}\right)=\sum_{k \in \mathbb{Z}} a_{i}[k] e^{-j 2^{i} \omega k}=2^{-i} \sum_{k \in \mathbb{Z}}\left|\hat{\varphi}_{i}\left(\omega+2 \pi k / 2^{i}\right)\right|^{2}$, where $a_{i}[k]=\left\langle\varphi_{i}(\cdot), \varphi_{i}\left(\cdot-2^{i} k\right)\right\rangle$ is the Gramor autocorrelation-sequence of the basis $\left\{\varphi_{i}\left(t-2^{i} k\right)\right\}_{k \in \mathbb{Z}} \cdot A_{i}(z)$, the $z$-transform of $a_{i}[k]$, is also referred to as the autocorrelation filter.

It can be proved that $\left\{\tilde{\varphi}_{i}\left(\cdot-2^{i} k\right)\right\}$ is a Riesz basis of $V_{(i)}$. The dual scaling filter is then obtained as

$$
\tilde{H}_{i}(z)=\frac{A_{i}(z)}{A_{i+1}\left(z^{2}\right)} H_{i}(z),
$$

and the dual two-scale relation is

$$
\tilde{\varphi}_{i+1}(t)=\sum_{k} \tilde{h}_{i}[k] \tilde{\varphi}_{i}\left(t-2^{i} k\right) .
$$

The wavelet function

$$
\psi_{i+1}(t)=\sum_{k} g_{i}[k] \varphi_{i}\left(t-2^{i} k\right)
$$

is obtained by solving the orthogonality relation

$$
\left\langle\psi_{i+1}(\cdot), \varphi_{i+1}\left(\cdot-2^{i+1} k\right)\right\rangle=0 .
$$

The general solution for $g_{i}[k]$ is given by

$$
G_{i}(z)=-z Q_{i}\left(z^{2}\right) H_{i}^{*}\left(-z^{-1}\right) A_{i}(-z),
$$

where the filter $Q_{i}(z)$ must be chosen to be bounded and non-vanishing on the unit circle. By imposing the perfect-reconstruction condition, we get the dual scaling filter as well,

$$
\tilde{G}_{i}(z)=-z \frac{\tilde{H}_{i}^{*}\left(-z^{-1}\right)}{A_{i}(-z) Q_{i}^{*}\left(z^{-2}\right)},
$$

where $\tilde{g}_{i}[k]$ are the coefficients in the dual wavelet relation

$$
\tilde{\psi}_{i+1}(t)=\sum_{k} \tilde{g}_{i}[k] \tilde{\varphi}_{i}\left(t-2^{i} k\right)
$$

The basis functions can also be orthogonalized to yield orthonormal wavelets. 


\section{PROPERTIES}

The new wavelet bases have the following remarkable properties, which may be useful in signal-processing applications:

Property 1. (Reproduction of null space). Suppose that $L_{\vec{\nu}}(s)$ has a zero $\alpha$ of order $\gamma$. Then, at each scale $i \in \mathbb{Z}$ and for $n=0, \ldots,\lceil\gamma\rceil-1$, the exponential monomial $t^{n} e^{\alpha t}$ has the scaling-function representation

$$
t^{n} e^{\alpha t}=\sum_{k \in \mathbb{Z}} p[k] \varphi_{i}\left(t-2^{i} k\right),
$$

where $p[k]$ are suitable coefficients.

As $\varphi_{i}$ satisfies the generalized Strang-Fix conditions, $t^{n} e^{\alpha t}$ belongs to the null space of the operator $\mathrm{L}_{\vec{\nu}}$. The property is then a direct consequence of the null-space reproduction property of the B-splines. More generally, any element $p(t)$ of the null space of $\mathrm{L}_{\vec{\nu}}$ can be expressed via the shifts of the scaling function. The orthogonality of the wavelet and scaling-function spaces then yields the following:

Property 2. (Vanishing null-space elements). Suppose that $L_{\vec{\nu}}(s)$ has a zero $\alpha$ of order $\gamma$. Then, for each scale $i \in \mathbb{Z}$, shift $t_{0} \in \mathbb{R}$, and degree $n=0, \ldots,\lceil\gamma\rceil-1$, the analysis wavelet satisfies

$$
\int_{-\infty}^{\infty} t^{n} e^{\alpha t} \tilde{\psi}_{i}\left(t-t_{0}\right) \mathrm{d} t=0
$$

In other words, the scalar product with the analysis wavelet $\tilde{\psi}_{i}$ makes vanish any element $p(t)$ of the null space of $\mathrm{L}_{\vec{\nu}}$.

\section{IMPLEMENTATION}

To perform the decomposition and the reconstruction in the new bases, we use an adapted version of Mallat's fast filterbank algorithm with scale-dependent filters. The filters $G_{i}(z), H_{i}(z), \tilde{G}_{i}(z), \tilde{H}_{i}(z)$ should be pre-calculated at each scale according to the formulas presented in Section 4.

To initialize the decomposition, we need to obtain the fine-scale $(i=0)$ projection coefficients $c[k]$ such that $f\left(k_{0}\right)=\sum_{k \in \mathbb{Z}} c[k] \varphi_{0}\left(k_{0}-k\right)$. We also need to calculate the autocorrelation filter $A_{0}(z)$, which will allow us to determine the coarser scale ones thanks to the recurrence relation:

$$
A_{i+1}\left(z^{2}\right)=\frac{1}{2}\left(A_{i}(z) H_{i}(z) H_{i}^{*}\left(z^{-1}\right)+A_{i}(-z) H_{i}(-z) H_{i}^{*}\left(-z^{-1}\right)\right) .
$$

The $c[k]$ 's can be obtained by prefiltering the function samples with $P(z)=\left(\sum_{k} \varphi_{0}(k) z^{-k}\right)^{-1}$.

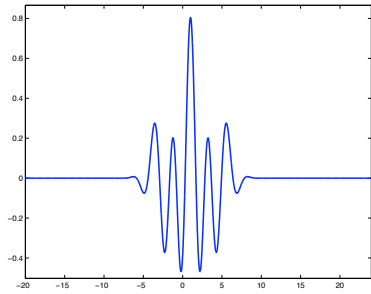

(a) Wavelet $\psi_{1}, i=1$

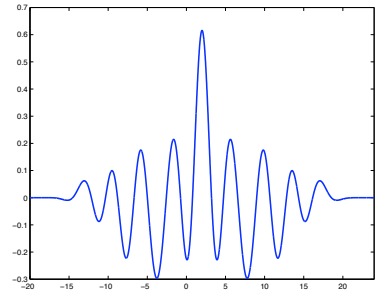

(b) Wavelet $\psi_{2}, i=2$

Figure 3. Wavelets at scales $i=1, i=2 ; \hat{L}_{\vec{\nu}}(\omega)=-\left(-\omega^{2}+\nu_{1}^{2}\right) /\left(\left(-\omega^{2}+\nu_{2}^{2}\right)^{2} \omega^{2}\right), \nu_{1}=5 j, \nu_{2}=5 \pi j / 8$. 


\section{EXAMPLES}

We illustrate the construction by choosing the operator with a rational transfer function $\hat{L}_{\vec{\nu}}(\omega)=-\frac{\left(\omega^{2}+\nu_{1}^{2}\right)}{\left(\omega^{2}+\nu_{2}^{2}\right)^{2} \omega^{2}}$ with $\nu_{1}=5 j$ and $\nu_{2}=5 \pi j / 8$. $\hat{L}_{\vec{\nu}}(\omega)$ has Hermitian symmetry, which ensures that the corresponding timedomain scaling functions and wavelets are real. In Figure 3, we show the wavelets $\psi_{1}$ and $\psi_{2}$ in the time domain; these have a strong oscillatory character, with a larger number of lobes as the scale gets coarser. It is clearly apparent that $\psi_{2}$ is not a dilate of $\psi_{1}$.

The plots in Figure 4 show the effect of the poles $\left( \pm \frac{5 \pi}{8} j, 0\right)$ and of the zeros $\pm 5 j$ on the frequency response of the scaling function and of the wavelet. In Figure $4(a)$, the pole $-\frac{5 \pi}{8} j$ produces a peak of the scaling-function

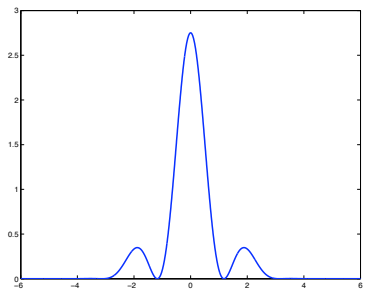

(a) Scaling-function spectrum $\left|\hat{\varphi}_{1}(\omega)\right|, i=1$

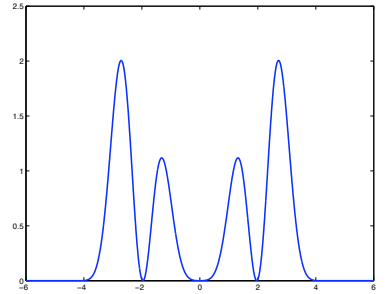

(b) Wavelet spectrum $\left|\hat{\psi}_{1}(\omega)\right|, i=1$

Figure 4. The spectrum of the scaling function and the spectrum of the wavelet; $\hat{L}_{\vec{\nu}}(\omega)=-\left(\omega^{2}+\nu_{1}^{2}\right) /\left(\left(\omega^{2}+\nu_{2}^{2}\right)^{2} \omega^{2}\right)$, $\nu_{1}=5 j, \nu_{2}=5 \pi j / 8$.

spectrum near $\omega=-\frac{5 \pi}{8}$ and makes it vanish with periodicity $\pi$. There is also a peak at the origin because of the pole at $\omega=0$. The zero at $-5 j$ makes the frequency response vanish at $\omega=-5$. In contrast, the wavelet spectrum (Figure $4(\mathrm{~b})$ ) vanishes at $\omega= \pm \frac{5 \pi}{8}$ and $\omega=0$, as a result of the vanishing null-space element property of generalized L-spline wavelets.

\section{CONCLUSION}

We have introduced a general procedure for constructing wavelet-like bases of $L_{2}$ based on a family of admissible operators $\mathrm{L}_{\vec{\nu}}$ of order $r$. Remarkably, the multiresolution spaces are specified by the transfer function $L_{\vec{\nu}}(s)$ of the operator. We have formulated the generalized Strang-Fix conditions that a scaling function must satisfy in order to reproduce the null space of a given operator L and have demonstrated that the generalized B-spline satisfies it. The constructed wavelets come in different flavors: basic (B-spline), dual, and orthonormal. They have the ability to kill the null space of $\mathrm{L}_{\vec{\nu}}$, which generalizes the notion of vanishing moment found in the conventional wavelet theory. One recovers the polynomial B-spline case by choosing $L_{\vec{\nu}}(s)=s^{N}$, where the corresponding operator is the $N$-th derivative.

The generalized L-spline wavelets are not dilates of a single function anymore; however, they still can be implemented using a scale-dependent version of Mallat's fast filterbank algorithm.

\section{ACKNOWLEDGMENTS}

This work is funded in part by grant 200020-101821 from the Swiss National Science Foundation.

\section{REFERENCES}

1. S. Mallat, A Wavelet Tour of Signal Processing, Academic Press, San Diego, 1998.

2. M. Unser and T. Blu, "Wavelet theory demystified," IEEE Trans. Signal Process. 51, pp. 470-483, February 2003.

3. M. Unser and T. Blu, "Fractional splines and wavelets," SIAM Review 42, pp. 43-67, March 2000. 
4. I. Khalidov and M. Unser, "From differential equations to the construction of new wavelet-like bases," IEEE Trans. Signal Processing, in press.

5. T. Lyche and L. L. Schumaker, "L-spline wavelets," in Wavelets: Theory, Algorithms, and Applications, C. K. Chui, L. Montefusco, and L. Puccio, eds., pp. 197-212, Academic Press, 1994.

6. M. Unser and T. Blu, "Generalized smoothing splines and the optimal discretization of the Wiener filter," IEEE Trans. Signal Process. 53, pp. 2146-2159, June 2005. 